\title{
Challenges experienced by nurses in the implementation of a healthcare reform plan in Iran
}

\author{
Shahin Salarvand ${ }^{1}$, Maryam Azizimalekabadi ${ }^{2}$, Azadeh Akbari Jebeli ${ }^{3}$, Mohamadreza Nazer ${ }^{4}$
}

\author{
${ }^{1}$ M.Sc. of Nursing, Faculty Member, Social Determinants of Health Research Center, Nursing and Midwifery \\ Faculty, Lorestan University of Medical Sciences, Khorramabad, Iran \\ ${ }^{2}$ MA in TEFL, Lecturer, English Language Department, Sheikhbahaee University, Isfahan, Iran \\ ${ }^{3}$ M.Sc. of Nursing, Lecturer, Psychiatric Nursing Department, Nursing and Midwifery Faculty, Isfahan University \\ of Medical Sciences, Isfahan, Iran \\ ${ }^{4}$ M.D. of Infectious and Tropical Disease Specialist, Associate Professor, Hepatitis Research Center, Medical \\ Faculty, Lorestan University of Medical Sciences, Khorramabad, Iran
}

\section{Type of article: Original}

\begin{abstract}
Introduction: The Healthcare Reform Plan is counted as a plan for improving healthcare services in Iran. Undoubtedly pros and cons can be seen either in plan or implementation. This study was conducted to describe nurses' challenges in implementing healthcare reform in Iran.

Methods: A qualitative method centered upon conventional content analysis was applied. We used purposive sampling and data saturation was obtained by 30 participants. Data were analyzed using MAXQDA software.

Results: Challenges experienced by nurses in the implementation of this reform include; unsuitable infrastructure, unfavorable vision, a complicated challenge, the necessity of monitoring, control plan outcomes, the impact on nurses, people's misconceptions and solutions.

Conclusions: The Healthcare Reform Plan in Iran is a solution to establish equality in the health system, however, to eliminate these challenges, revision and appropriate foundation of infrastructures is called for.
\end{abstract}

Keywords: Health Care Reform Plan, Nursing, Iran

\section{Introduction}

Health systems are not only responsible for promoting the health of a community but are also in charge of people's expectations, as the evolving needs of society make health system reform inevitable throughout the world (1). The Iranian government has put several more reforms into practice such as family physician, merging health services and medical education, the policy of autonomy of hospital programs and health system reform plan, the last reform, triggered in May 2014, is the most recent reform of health system in Iran (2). Before the implementation of the healthcare reform plan, several studies indicated a lack of equality in accessibility to standardized health services and high payment out of pocket expenditure as a challenge in Iran's health system, and have offered some solutions $(1,2-4)$. Also, more than $50 \%$ of the expenditures have been out of pocket. The percentage for direct financial payment for medical care expenditures resulted in disastrous healthcare expenditures for $2.5 \%$ of the Iranian population and eventually caused the poverty of $1 \%$ of the population annually (3). Due to necessity, health authorities of our country felt these problems and decided to implement a plan of health system reform, This plan consisted of seven axes including decreasing the payments of the hospitalized patients in affiliated hospitals to the health ministry, supporting the inhabitancy of physicians in deprived areas, presence of resident physicians in public hospitals, promoting the quality of visit services in the hospitals, promoting the quality of hoteling in hospitals, financial support for the incurable, specific and needy patients, development of normal delivery and supervision on performance of the plan (5). As no thought or plan is out of deficiency, undoubtedly pros and cons can be seen either

\section{Corresponding author:}

Associate Professor Dr. Mohamadreza Nazer, Hepatitis Research Center, Medical Faculty, Lorestan University of Medical Sciences, Khorramabad, Iran.

Tel: +98.9167104954, Fax: +98.6633120150, Email: mohammadia1394@gmail.com

Received: October 11, 2016, Accepted: January 20, 2017, Published: April 2017

iThenticate screening: January 01, 2017, English editing: March 04, 2017, Quality control: April 02, 2017

(C) 2017 The Authors. This is an open access article under the terms of the Creative Commons Attribution-NonCommercialNoDerivs License, which permits use and distribution in any medium, provided the original work is properly cited, the use is non-commercial and no modifications or adaptations are made. 
in plan or implementation, and all the essential corrections should be provided and deficiencies should be removed (6). Unquestionably, what guarantees the successful implementation of the plan is paying special attention to internal customers i.e. healthcare providers in the public sector (7), and nurses as the biggest group of healthcare professionals that provide care in different health centers and have an ideal status to answer the numerous challenges of the health and treatment system (8). It is essential that nurses' perceptions, as key members of the health care team be investigated, as one side of the quality is under the influence of observers and audiences who interpret the quality and define it based on their own point of view. Moreover, one of the implications of qualitative research is to gain information in areas where the body of knowledge is not sufficient. For this reason, content analysis was selected as it is a suitable and pliable method for expanding cognition of the participants' experiences (9). This study was conducted to describe nurses' challenges in implementing a healthcare reform plan in Iran

\section{Material and Methods}

\subsection{Participants and Data Gathering}

A qualitative method centered upon conventional content analysis was applied. Participants were purposively selected from nursing societies of Isfahan, Khorramabad, Mashhad, Tehran, Babol and Najafabad. Qualitative sampling may start with volunteer informants and may be continued with new participants through snowballing (10). In this study, we started sampling with volunteer informants and continued with new participants through snowballing of enriched informants with sampling diversity of different locations. Data saturation was obtained by 30 participants. Semi-structured interviews lasted between 40 to 90 minutes. Central question of the interviews was "What are your experienced challenges of implementing the healthcare reform plan?" and follow-up questions were asked to come to more profound information.

\subsection{Ethical Considerations}

The University ethics committee (LUMS.REC.1395.152) approved this study. Informed consent of participants was also obtained and participants were completely aware of the goals and method of the study consisting of the necessity of the recording of the interview and also their own rights i.e. confidential information, anonymity and their right to cease cooperation.

\subsection{Data Analysis}

Data were collected and analyzed simultaneously. Thus, after the end of every interview, recorded participants' statements were listened to carefully and transcribed verbatim. Transcripts were studied several times to come to a better understanding of participants' feelings and experiences. Then meaningful information was underlined and important sentences were highlighted. Next, it was attempted to extract a concept indicating the essential part of the individual's thought. Thus, those codes which had conceptual similarity were fit under one category. Moreover, primary categories were merged and more comprehensive categories with more abstract concepts were formed. And finally, certain categories with higher abstract levels were made and their interrelationships were determined. As an example, to mix several codes such as physical, mental and professional outcomes, they were put under a more general category called the impact on nurses. As bracketing in data analysis process, researcher's preconception and prejudgments were put aside. MAXQDA software was applied for data analysis.

\subsection{Trustworthiness}

For evaluating trustworthiness, four categories i.e. credibility, conformability, transferability and dependability were applied. Credibility was put into practice by prolonged engagement with participants for one year and data collection was done by maximum diversity, member checking and peer checking. Participants were chosen from different hospitals with different services and environments. Furthermore, they were in different ages, job experiences, education and organizational levels. Also, the researcher sent the findings and extracted codes to participants to have them validated by their acceptance. The findings and extracted codes of the study were also sent to a qualitative research expert and the trend of research and categories were confirmed. The researcher explains the procedure of the research in detail to increase dependability and explain how to achieve the results to help other researchers in understanding of the process. In the present study, confirmability is achieved through a comprehensive report of the researchers' activities, analyses and textual clips from interviews for every category. The research team attempted to register and report research phases and take decisions so that if others had tendency to follow the process, inquiry audit would be possible. In this study, to help the transferability, sampling of the participants' demographic information was explained. It was also attempted to increase the transferability by providing appropriate quotations. 


\section{Results}

Participants of this study consisted of 30 nurses i.e. 12 clinical nurses, 10 nursing managers and 8 academic members of nursing. After data analysis, 450 primary codes and 8 principal categories appeared that consisted of: unsuitable infrastructure, unfavorable vision, a complicated challenge, the necessity of monitoring, plan outcomes, the impact on nurses, people's misconceptions and solutions (Table 1).

Table 1. Categories and sub categories resulted of data analysis.

\begin{tabular}{|l|l|}
\hline Category & Subcategory \\
\hline \multirow{4}{*}{ Unsuitable infrastructure } & Lack of space and facilities \\
\cline { 2 - 2 } & Lack of creating culture before implementing the plan \\
\cline { 2 - 2 } & Nurse insufficiency \\
\hline \multirow{2}{*}{ Anfavorable vision } & Unsuccessful plan \\
\cline { 2 - 2 } & Failure economically and financially \\
\hline \multirow{2}{*}{ The necessity of monitoring } & The appearance of problems in other areas \\
\cline { 2 - 2 } & Failing to achieve the predetermined goals \\
\cline { 2 - 2 } & Conitoring and controlling physicians' performance \\
\hline Plan outcomes & Positive outcomes of plan \\
\hline \multirow{3}{*}{ The impact on nurses } & Negative outcomes of plan \\
\cline { 2 - 2 } & Physical, mental, social and professional outcomes \\
\hline \multirow{2}{*}{ People's misconceptions } & Trficiency in care duties \\
\hline \multirow{2}{*}{ Solutions } & Lack of knowledge toward nursing profession \\
\cline { 2 - 2 } & Negative perceptions about nurses due to faced problems \\
\hline & Supervision \\
\cline { 2 - 2 } & Giving value to nurses' work and human values \\
\cline { 2 - 2 } & Trying to revise the plan \\
\hline
\end{tabular}

\subsection{Unsuitable infrastructure}

This category contains three subcategories; lack of space and facilities, lack of creating culture before implementing the plan and nurse insufficiency. Participants of this study pointed to the planning and unsuitable infrastructures before implementing the plan. Participant 14 commented on social and communication problems due to lack of creating culture before implementing the plan, and lack of proper performance of the plan: "Weaknesses of the plan can be counted as lack of suitable infrastructure for starting the plan, ignoring suitable beds to perform the plan and providing facilities like space, human resources and generally facilities both qualitatively and quantitatively". (P: 14) "If it was well-structured at the beginning, and the society was well-prepared for its implementation and people's culture was upgraded, it would be a good plan". (P: 11)

\subsection{Unfavorable vision}

There were two subcategories in this category; unsuccessful plan and failure (both economically and financially). Participants did not have a good perspective about the future of the plan. "In my opinion, it is not a successful plan as patients will suffer and also as utilized equipment may not have a good quality. Also, the government may face failure economically and financially". (P: 18)

\subsection{A complicated challenge}

This category contained the appearance of problems in other areas, failing to achieve the predetermined goals of this plan. Participants' statements indicate a complicated challenge which is difficult to be solved. They argued the reverse outcomes and failing to achieve the predetermined goals of this plan. "This plan has changed the direction of problems to another side and by their reform, comprehensive results will be achieved. If increasing the number of patients decreases the quality of the service, the purpose of this plan will not be achieved and new problems will emerge". (P: 23) Referring the patients to private centers to get more qualitative services or diagnostic equipment and increasing patients' treatment expenditures are other challenges which are related to inappropriate planning. "I remember an experience related to offering diagnostic services and increasing patient's treatment expenditures. It was said that in other centers, reservation service is not good or necessary service is not provided in public centers 
and this will cause patients' dissatisfaction and will make the families criticize the health professional workers without regarding the fact that they are not culpable". (P: 12)

\subsection{Necessity of monitoring and control}

This category comprised two subcategories; the necessity of monitoring and controlling physicians' performance and controlling private hospitals. "It is a positive plan provided that it is monitored well. This plan could be successful if physicians' performance and private hospitals were also controlled". "This plan could be successful if private hospitals were also controlled, as in the current situation, surgeons take their own patients to private hospitals and it is detrimental for people and public hospitals to accept less patients". (P: 9)

\subsection{Plan outcomes}

\subsubsection{Positive outcomes of plan}

Participants referred to improving the physical environment of hospitals and their equipment, people's satisfaction with receiving insurance pamphlets, patients' current satisfaction, presence of resident physicians and increasing the presence of specialist physicians in hospital, Patients' satisfaction due to decreasing treatment expenditure in public centers and patients' satisfaction due to not paying informally. "Considering the fact that physicians are not allowed to receive informal payments, they send most of their own patients to private centers and finally gain their own considered profit. Also, patients are satisfied with this plan as they are not forced to pay a lot of expenditures". (P: 17) Participant 21 commented on the positive points of the plan, such as providing patients with needed medicines and equipment without wasting time.

\subsubsection{Negative outcomes of plan}

Participants referred to nurses' dissatisfaction with this plan, decreasing the quality of health services, increasing the number of patients during nursing force shortage, nurses' unrevised payment for increased workload, unequal payment, inequality between physicians and nurses, extreme patient-centeredness, weakening the role of the nurses in the society, nursing isolation, nurses' fatigue, demotivation, physicians and patients' insolence toward nurses, patients' threat of complaining about the nurses, physicians' disobedience in terms of shifting the patients to private hospitals. This study found that the quality of treatment services has decreased due to physicians' abandonment of public hospitals and centers. Accordingly, patients go to private hospitals to receive high quality services. "Most of the physicians left the public hospitals as they didn't receive informal payments; therefore, patients didn't have access to competent physicians or better care, they were forced to go to private hospitals". (P: 18)

\subsection{The impact on nurses}

This category consisted of the physical, mental, social and professional outcome on nurses and also, efficiency in care duties and trying to cope with the plan. Participant 12 referring to working compulsory overtime due to nursing staff shortage of this plan said: 'In my opinion, this plan has decreased nurses' status more than before, as when people go to hospital, they consider nurses responsible for failures or procedures and quickly share the problem in social media and it has ruined nurses' status more so than before". (P: 9) "Nurses' positions have not changed in this plan and they continue to do what they did in the past, but with a greater working load". (P: 5). "Since this plan is considered free for people, they have found more tendency to go to hospitals and this rush makes the nurses tired and has negative effects on them".(P: 30) From participants' view, performing this plan has decreased care quality. "Taking care of the patient has decreased due to extreme bureaucracy, and most of the nurses' time is devoted to filling out the documents...". (P: 21) “... Most of the nurses went to non-medical centers or resigned”. (P: 9) "Authorities and decision makers must consider that mental problems of the active groups in treatment and health sectors will influence the quality of their performance, and finally, these are people and patients who will be deprived of desirable service qualities". (P: 8) Participants have observed and experienced nurses' tolerance and their attempt to maintain care quality. "The nursing community is so understanding and continues its own duties in terms of serving people and patients in the best possible way. Yet, they will lose their motivation over time". (P: 16) On the other side, this plan has made nurses update and increase their scientific advance.

\subsection{People's misconceptions}

In this category, participants have pointed to people's lack of knowledge toward the nursing profession and people's negative perceptions about nurses due to faced problems "Patients' unawareness about the problems that come from this plan has made a lot of challenges". (P: 18) “...in emergency wards that mostly outpatients refer, when we ask them to get the bill of procedure, they become angry and say surprisingly: isn't it free? So, what are the materials talked in TV? and in most of the shifts, we face this problem and we should explain it to people". (P: 26) 


\subsection{Solutions}

This category contained three subcategories; supervision, giving value to nurses' work and human values, trying to revise the plan. Participants stated; considering job difficulties, creating the culture in the society, facing culpable physicians, making enough budget for hospitals to provide equipment, considering work sequence, increasing nurses' salary proportionate to their job difficulty, considering the proportion between the number of patients and nurses, having control on private hospitals, decreasing surgery in private hospitals, correct implementation and considering the treatment cadre. "This plan needs revision in order to remove the deficiencies". (P: 15) "This plan has achieved some of its goals but hasn't achieved others and has had outcomes that are noteworthy”. (P: 7)

\section{Discussion}

This study was aimed to describe nurses' challenges toward implementing a healthcare reform plan in Iran. In the present study, participants referred to unsuitable infrastructures like lack of space and facilities, and nurse insufficiency. The results of Gharibi et al. are consistent with this study that infrastructure has not been prepared for the successful implementation of this plan (5). Undoubtedly, every evolution needs prediction and infrastructure provision to be performed well in order to have an acceptable effectiveness. Participants did not have a favorable vision about the plan and mostly predicted that it will face failure. A study by Heshmati proves that in the first year, the plan's vision was desirable due to decreasing hospitalized patients' expenditures and increasing healthcare providers' salary. However, some challenges will have negative effects on this health reform plan in future, like financial load for government, ignoring primary healthcare and inefficient payment methods, low financial resources, medical specialists' inequitable distribution, disregarding the outpatients in general departments and hospitalized patients in private hospitals (2) In contrast, several studies stated that the healthcare reform plan has been successful in Iran $(11,12)$. In this study, participants have experienced complicated challenges like the appearance of problems in other areas and failing to achieve the predetermined goals. With regard to the increased workload of nurses, nurse shortage is really challenging and important in Iran (8) and could result in low quality of care. Decreasing medical services quality and non-commitment of some physicians to provide optimal care due to omitting informal payment were other challenges mentioned in Nekoeimoghadam's study (12). A study by Gholami found the following problems related to the healthcare reform plan implementation; increasing working load followed by decreasing work quality, insufficient equipment and space, insufficient staff, unrevised salary of nurses and staff, increasing the number of visits and insufficient information to support and evaluate the reform plan to determine the real effects of the plan (13). Participants referred to positive and negative outcomes of the plan. Positive outcomes including improving the hospital's physical environment and hoteling quality are consistent with findings of a study by Gharibi et al., highlighting that addressing hoteling has been improved (5). Given that hoteling development can improve hospital image, it will be satisfactory to patients and their families. Another positive outcome was counted as the presence of resident physicians and increasing the presence of specialists in hospital, that were consistent with a Gharibi et al. study as the strength of the reform plan and it required the hospitals to have the main specialties (5). Present study found, physicians' relative satisfaction from the plan. Other studies pointed out that the healthcare reform plan has had positive effects on physicians' job satisfaction (15, 14). Patients' satisfaction due to decreasing out of pocket payment in public centers has been one of the positive outcomes of this plan, and is expressed and observed exceedingly in literature, and has been one of the plan goals which was almost achieved (15-17). In the present study, participants also pointed to nurses' dissatisfaction with the plan, damaging the provision of quality health services as one of the dimensions of the reform plan which has a negative outcome. Increasing work load, unchanged salaries and staff insufficiency were the main factors that decreased hospital staff's satisfaction in terms of health reform implementation (13). In addition, Participants said, the fact that patients are referred to private centers to receive high quality services and due to sense of profiting by some physicians, any related study with this subcategory was not found. Participants pointed to the impact of plan implementation on nurses with subcategories like physical, mental, social and professional outcomes, care quality outcomes and trying to cope with the plan. Nursing participants of this study did not feel they were valuable or respected. Gharibi et al. found physicians and residents didn't have positive interaction with their colleagues especially with nurses and ordered intolerably, and it has made nurses fear of physicians and be wary of their inappropriate behavior (5). Moreover, the implementation of this plan has intensified the causes of burnout for nurses. Mirfarhadi's findings also showed this will lead to job exhaustion and result in low quality services (18). This study showed that nurses attempted to adjust to the induced problems of the plan by maintaining care quality and updating their own scientific information and responsiveness to patients. Other studies found that a high level of patients' satisfaction of nursing care services and responsiveness have been promoted in this plan (19). Participants of this study expressed the necessity of plan supervision and having more control on physicians' performance and the private sector. The Healthcare Reform Plan has no influence on physicians' autonomy. However, inappropriate 
behavior of some physicians cannot mistakenly be considered autonomous. In Gharibi's study, some deficiencies were found in terms of improving visit quality such as physicians' self-discipline towards their own clinical behavior, lack of government supervision or responsible institutions on their performance (5). This study indicated the necessity of controlling private hospitals, any related study with this subcategory was not found. People's misconceptions was another category found in this study and also knowing nurses as culpable for the problems induced from the plan, caused more pressure on nurses. Farsi's study showed that nurses in Iran and indeed around the world have some common challenges such as the weak social position of nurses (20). It could be due to lack of knowledge toward the nursing profession, any related study with this subcategory was not found. Participants pointed to some possible solutions to solve the plan's problems. Several studies stated solutions such as coordinated supervision from all dimensions of the health system in order to implement the evolution effectively should be considered and infrastructures, equipment and facilities, human resources, financial resources, health and treatment sectors and appropriate distribution of sources should be revised in implementing the healthcare reform plan (3). If nurses work where they are able to benefit their knowledge and proficiency, they will be allowed to make decisions in their working area and take managerial responsibilities; therefore, their self-worth will increase in an organization and they will probably have higher job satisfaction (18).

\section{Conclusions}

At this point of time, we can better evaluate and analyze this plan because its weaknesses have been revealed and have made its evolution inevitable. This study found that nurses were satisfied with the purpose of the Healthcare Reform Plan. However, some factors had made them dissatisfied with the plan such as: nurse shortage, insufficient equipment and facilities and inappropriate behavior of physicians. It is necessary to revise and evaluate several aspects of the plan.

\section{Acknowledgments:}

We give special thanks to the Vice Chancellor of Research of Lorestan University of Medical Sciences for financial support of this research. Also, we are grateful to all the nurses who participated in this study.

\section{Conflict of Interest:}

There is no conflict of interest to be declared.

\section{Authors' contributions:}

All authors contributed to this project and article equally. All authors read and approved the final manuscript.

\section{References:}

1) Daneshkohan A, Karami M, Najafi F, Matin BK. Household catastrophic health expenditure. Iranian journal of public health. 2011; 40(1): 94. PMID: 23113061, PMCID: PMC3481728.

2) Heshmati B, Joulaei H. Iran's health-care system in transition. The Lancet. 2016; 387(10013): 29-30. doi: 10.1016/S0140-6736 (15)01297-0. PMID: 26766344.

3) Manenti A. Health situation in Iran. Medical Journal of the Islamic Republic of Iran. 2011; 25(1): 1-7.

4) Hajizadeh M, Nghiem HS. Hospital care in Iran: an examination of national health system performance. International Journal of Healthcare Management. 2013; 6(3): 201-10. doi: 10.1179/2047971913Y.0000000042.

5) Gharibi F, janati A, Farajollah Beiknoori M, Amini Daghalian B. A Survey of Health System Reform Circumstances from the Experiences of Managers and Nurses of Tabriz Taleghani Hospital. Depiction of Health. 2015; 6(1): 1-10.

6) Witter S, Toonen J, Meessen B, Kagubare J, Fritsche G, Vaughan K. Performance-based financing as a health system reform: mapping the key dimensions for monitoring and evaluation. BMC Health Serv Resv. 2013; 13(1): 367. doi: 10.1186/1472-6963-13-367. PMID: 24073625, PMCID: PMC3849795.

7) Akhondzadeh R. Health system transformation project, an opportunity or a threat for doctors. JAP. 2014; 5(1): 1-2.

8) Zarea K, Negarandeh R, Dehghan - Nayeri N, Rezaei - Adaryani M. Nursing staff shortages and job satisfaction in Iran: Issues and challenges. Nursing \& health sciences. 2009; 11(3): 326-31. doi: 10.1111/j.1442-2018.2009.00466.x.

9) Graneheim UH, Lundman B. Qualitative content analysis in nursing research: concepts, procedures and measures to achieve trustworthiness. Nurse education today. 2004; 24(2): 105-12. doi: 10.1016/j.nedt.2003.10.001. 
10) Polit DF, Beck CT. Nursing research: Generating and assessing evidence for nursing practice. 9th ed: Lippincott Williams \& Wilkins; 2012.

11) Moradi-Lakeh M, Vosoogh-Moghaddam A. Health Sector Evolution Plan in Iran; equity and sustainability concerns. International journal of health policy and management. 2015; 4(10): 637 . doi: 10.15171/ijhpm.2015.160.

12) Nekoeimoghadam M, Esfandiari A, Ramezani F, Amiresmaili M. Informal payments in healthcare: a case study of Kerman province in Iran. International journal of health policy and management. 2013; 1(2): 157. doi: 10.15171/ijhpm.2013.28.

13) Gholami S, Oveisi S, Ghamari F, Etedal MG, Rajaee R. Study of Educational Hospital Employees' Satisfaction with the Administration of the Health Reform Plan in Ghazvin, 2015. Electron physician. 2015; 7(7):1500. doi: 10.19082/1500. PMID: 26767104, PMCID: PMC4700896.

14) Aasland OG, Rosta J, Nylenna M. Health care reforms and job satisfaction among doctors in Norway. Scand J Public Health. 2010; 38(3): 253-8. doi: 10.1177/1403494810364559. PMID: 20215483.

15) Sajjadi H, Zaboli R. An Assessment of the Positive Effects of Health Reform Plan Implementation from the Perspective of Hospital Directors. Health Inf Manage. 2016; 13(1): 55-60.

16) Goudarzian AH, Sharif Nia H, Jafari H, Jamali S, Badiee M, Sayemi Z, et al. Inpatient Satisfaction with Health System Transformation Project in Mazandaran Educational Hospitals, Iran. Journal of Mazandaran University of Medical Sciences. 2016; 26(136): 190-5.

17) Heidarian N, Shagayeg V. the effect of health system reform on patients' out of pocket in public hospitals in Isfahan. Journal of medical council of islamic republic of iran. 2015; 33(3): 187-94.

18) Mirfarhadi N, Moosavi S, Tabari R, Kazemnejad E. Predictive factors of job satisfaction among nurses in therapeutic-educational centers of Guilan University of Medical Science. Journal of Neyshabur University of Medical Sciences. 2014; 2(4): 27-35.

19) Piroozi B, Moradi G, Nouri B, Mohamadi Bolbanabad A, Safari H. Catastrophic health expenditure after the implementation of health sector evolution plan: a case study in the west of Iran. Int J Health Policy Manag. 2016; 5(7): 417-23. doi: 10.15171/ijhpm.2016.31. PMID: 27694669, PMCID: PMC4930347.

20) Farsi $Z$, Dehghan - nayeri N, Negarandeh R, Broomand S. Nursing profession in Iran: an overview of opportunities and challenges. Jpn J Nurs Sci. 2010; 7(1): 9-18. doi: 10.1111/j.1742-7924.2010.00137.x. PMID: 20618672. 